\title{
Evaluation of objective and subjective indicators of death in a period of one year in a sample of prevalent patients under regular hemodialysis
}

\author{
Paulo R Santos
}

\begin{abstract}
Background: To identify objective and subjective indicators of death in prevalent hemodialysis (HD) patients in a follow-up study of 12 months.

Methods: The study included end-stage renal disease patients undergoing HD and analyzed demographic and laboratory data from the dialysis unit's records. Baseline data concerning socioeconomic status, comorbidity, quality of life level, coping style and depression were also assessed. For variables that differed in the comparison between survivors and non-survivors, Cox proportional hazards for death were calculated.

Results: The mortality rate was $13.0 \%$. Non-survivors differed in age, comorbidity, inclusion on the transplant waiting list and physical functioning score. The hazard ratios of death were 8.958 (2.843-28.223; $p<0.001)$ for comorbidity, $3.992(1.462-10.902 ; p=0.007)$ for not being on the transplant waiting list, $1.038(1.012-1.066 ; p=$ $0.005)$ for age, and $0.980(0.964-0.996 ; p=0.014)$ for physical functioning.
\end{abstract}

Conclusions: Comorbidity, not being on the transplant waiting list, age and physical functioning, which reflects physical status, must be seen as risk indicators of death among patients undergoing HD.

Keywords: End-stage renal disease, Hemodialysis, Mortality, Quality of life, Depression, Coping

\section{Background}

Fifty years ago the introduction of the Scribner shunt allowed the realization of repeated hemodialysis (HD) sessions [1]. This advance greatly extended the survival of patients suffering from end-stage renal disease (ESRD). Today there are some 80,000 ESRD patients undergoing HD in Brazil [2]. But the efficiency of dialysis treatment is limited. HD does not perform all normal kidney functions, and patients under HD retain several molecules, provoking uremic toxicity, oxidative stress and chronic inflammation [3]. The main consequence is high mortality. Mortality rates vary among HD patients throughout the world. It is low in Japan (6.6\%), intermediate in Europe (15.6\%) and Brazil (17.0\%), and high in the United States (21.7\%) [2,4]. Demographic and clinical characteristics, access to kidney transplantation and practice patterns explain the mortality differences.

Correspondence: prsantos@fortalnet.com.br

Sobral School of Medicine, Federal University of Ceará, Avenida Comandante Maurocélio Rocha Pontes, 100 CEP 62.042-280, Sobral, Ceará, Brazil
Age seems to be a good indicator of risk of death. In the Brazilian population, crude mortality is different according to age ranges, being $8.3 \%$ in the range between 20 and 39 years old and 59.0\% among patients above 60 [2]. Directly linked to age is comorbidity. Age and the most common ESRD etiologies, namely hypertension and diabetes, lead to multiple organ dysfunctions, mainly cardiovascular. However, samples of HD patients are not homogeneous. Roughly speaking, in underdeveloped areas the profile is younger patients with low comorbidity, with the principal etiology being glomerulonephritis rather than diabetes. In contrast, in developed areas there is prevalence of older and diabetic patients. Based on data showing a higher risk of death in earlier stages of chronic kidney disease than after reaching advanced stages and the requirements for dialysis [5], we can hypothesize that the main attention on infectious diseases in underdeveloped areas relegates medical assistance to patients with chronic diseases to secondary importance. Probably diabetics in 
underdeveloped regions without priority in medical assistance tend to die more than in developed areas before developing ESRD. Also, in underdeveloped areas life expectancy is lower, thus decreasing the emergence of diabetes.

Sobral is a city located in the semiarid region of northeast Brazil. Our unit in Santa Casa Hospital is the only renal unit within a radius of $200 \mathrm{~km}$. We serve a region of 1,800,000 inhabitants. As stated previously, due to the sample characteristics, mortality rates and predictors can vary. Virtually all our patients have low socioeconomic status [6]. Such patients need more social support to undergo dialysis. In Sobral, we provide living quarters and meals during the periodic dialysis sessions.

Most studies in Brazil are performed in more developed areas of the country, where the leading universities are located. The patient profile and mortality risk differ widely in the various regions of Brazil, depending on socioeconomic and demographic characteristics of the sample. Mortality indicators are mainly objective, like age, comorbibity and albumin level. However, quality of life has been increasingly recognized as an independent indicator of mortality [7]. So, the aim of this study is to identify objective and subjective indicators of death in prevalent HD patients from a renal dialysis unit located in a low-income area in a 12-month follow-up study.

\section{Methods}

\section{Sample}

The sample included ESRD patients undergoing HD during April 2009 in the only renal unit in northern Ceará state, northeast Brazil. The criteria for inclusion were age older than 18 , at least three months on dialysis and no previous transplantation. Out of 191 patients being treated by the unit that month, 161 were included. The reasons for exclusion were: 14 with less than three months on therapy, 8 with previous transplants, 5 refusals and 3 under 18 years old. All patients were undergoing conventional HD with polysulfone dialyzers (maximum number of reuses $=12$ ). The study protocol and informed consent form were approved by the ethics committee of Vale do Acaraú University, which is the only ethics committee in our region.

\section{Measurement of quality of life}

The measurement tool was the validated Brazilian version of the Medical Outcomes Study 36-Item Short Form Health Questionnaire (SF-36) [8]. The questionnaire was applied through interviews during April 2009, conducted by three professionals who did not belong to the dialysis unit team. This is a well-validated 36-item questionnaire covering issues relating to physical, psychological and social functioning. It generates scores from 0 (worst) to 100 (best) for eight sub-scales of quality of life: physical functioning, role-physical, bodily pain, general health, vitality, social functioning, roleemotional and mental health.

\section{Coping}

The first version of the Jalowiec Coping Scale [9] was used, adapted to Portuguese by Souza-Talarico and colleagues [10], which consists of 40 items to determine coping style. This is a well-validated instrument, based on the cognitive theory of psychological stress and coping proposed by Lazarus and Folkman [11]. The 40 items are grouped into two styles: problem-oriented coping, comprising 15 items (scores ranging from 15 to 75), and emotion-oriented coping, comprising 25 items (scores ranging from 25 to 125). Problem-oriented coping aims to make direct changes in a stressful situation, whereas emotion-oriented coping seeks to ameliorate emotions associated with the problem. Subjects are evaluated about coping styles according to a five-point Likert scale, ranging from "never do" to "always do".

\section{Depression}

For this analysis, the 10-item version of the Center for Epidemiologic Studies Depression Scale [12] was used. Respondents rate items by recalling the past week and using a three-point response scale, with higher scores indicating the presence and persistence of symptoms. A score ranging from 0 to 30 is calculated by summing the score of each item. A score $\geq 10$ is classified as depression.

\section{Patient data}

The demographic data, time on dialysis, inclusion on transplant waiting list and underlying etiology of ESRD were obtained from dialysis unit records. The underlying kidney disease was classified by clinical criteria and not by histopathology. Classification of socioeconomic status was according to criteria of the form issued by the Brazilian Association of Research Institutes [13]. This validated instrument is used in marketing surveys and population censuses and grades socioeconomic status into five subgroups: A (best status) through $\mathrm{E}$ (worst status). Besides income level, its criteria include educational level of the head of household and ownership of household appliances. Each patient was assigned a low, medium or high risk index based on comorbidity, as described by Khan et al. [14]. Khan's comorbidity index takes into consideration age in three classes and nine comorbidities: diabetes, myocardial infarction, angina pectoris, congestive heart failure, liver cirrhosis, obstructive pulmonary disease, systemic collagen disease, pulmonary fibrosis and visceral malignancies. The laboratory results were those routinely measured in HD 
patients: creatinine, albumin, hemoglobin, calcium and phosphorus and $\mathrm{Kt} / \mathrm{V}$. Kt/V was estimated using a second-generation Daugirdas formula [15].

The instruments SF-36, Jalowiec Coping Scale and the Center for Epidemiologic Studies Depression Scale were applied on the day of the patient's regular hemodialysis, before the dialysis session, in a private office by an interviewer who was not a renal unit staff member. After gathering the baseline data, we obtained data from the patients' medical records on death in the next 12 months (April 2009 through March 2010).

\section{Statistical analyses}

Comparisons between survivors and non-survivors were performed by the Fisher and Student's t tests, after using the Shapiro-Wilk test to verify if the data were normally distributed. Cox proportional hazards were calculated to measure the risk of death according to the variables that differed in the comparison between survivors and non-survivors. Censoring occurred at the end of one year of follow-up or renal transplantation. There was no other kind of loss besides transplantation.

\section{Results}

More than $70 \%$ of the patients were classified as pertaining to the lowest socioeconomic classes (D and E). Moreover, the majority of them had low comorbidity, and based on mean laboratory values, they presented a good level of albumin (which is an indicator of nutritional and inflammatory status). They also had good control of calcium-phosphorus product (levels above 55 $\mathrm{mg}^{2} / \mathrm{dl}^{2}$ increase mortality risk) and were submitted to an adequate dialysis dose, as estimated by the $\mathrm{Kt} / \mathrm{V}$ index (target $>1.2$ ). Nevertheless, the control of anemia was far from the ideal (mean hemoglobin of the sample was $8.5 \pm 1.7 \mathrm{~g} / \mathrm{dl}$ versus the recommended target of 11 to $12 \mathrm{~g} / \mathrm{dl}$ ). (Table 1 )

The most affected dimensions of QOL were role-physical and general health, and the best-scored dimensions were social functioning and bodily pain. There were $8 \%$ of depressed patients. From a possible maximum score of 75 related to problem-oriented coping, the mean score was $51.3 \pm 8.4$, and from a possible maximum score of 125 related to emotion-oriented coping, the mean score was $68.2 \pm 9.7$, showing a slight trend to more common use of problem-oriented coping. (Table 2)

The mortality rate was $13.0 \%$. Patients who died were older (53.6 \pm 11.9 vs. $43.1 \pm 15.7$ years; $p=0.002)$, had higher comorbidity ( $19.0 \%$ vs. $2.1 \%$; $p=0.002)$, were less often on the transplant waiting list $(23.8 \%$ vs. $57.1 \%, p=$ $0.005)$, needed more hospitalization (57.1\% vs. $7.1 \%$; $p<$ 0.001 ) and presented lower physical functioning scores $(42.6 \pm 26.3$ vs. $57.6 \pm 24.9 ; p=0.023)$. The comparisons of the characteristics between survivors and non-survivors are shown in Tables 1 and 2 .

Table 3 shows the hazard ratios of death according to each variable that differed between survivors and nonsurvivors.

\section{Discussion}

As previously stated, our sample is characteristic of an underdeveloped area, comprising young patients (mean age of 44.5 years) and few diabetics-only $9.9 \%$ of the sample. Our main interest was to test if emerging subjective measures, like quality of life, depression and coping, were able to predict death in a small sample like ours. But we found that traditional objective variables, mostly reflecting physical status, were the only indicators of death.

Measures of subjective feelings are validated as prognostic markers among HD patients [16-19]. But none of the subjective measures obtained from the sample correlated with risk of death, except one dimension of quality of life. However, this dimension correlated with risk of death was physical functioning, which is typically related to physical status. Its score is generated from questions about daily activities that become difficult because of the patient's poor health. Thus, physical functioning assessed by SF-36 seems to reflect physical status more than subjective feelings.

Overall, we found that the main difference between survivors and non-survivors can be summarized by physical status, assessed in our study by different variables. The question of colinearity between these variables must be emphasized. Age, comorbidity grade, not being in the transplant waiting list, and physical functioning, as assessed by SF-36, all summarize a single clinical condition: physical status. Age, comorbidity and physical functioning can be easily identified as correlated with physical status. Also, not being on the waiting transplant list reflects physical status due the fact that the main reasons for not being on the list are physical aspects linked to organ dysfunction.

Our results indicate that assessment of physical status is a valid way to stratify risk of death among HD patients even in small unicentric samples. Fortunately, physical status can be assessed in many easy ways, such as use of routine variables present in renal units' records, like age and not being on the transplant waiting list, and also through application of validated instruments. There are several kinds of instruments for clinical use to assess physical status which were extensively used in dialysis studies: (i) quality of life measurements: all generic instruments to measure quality of life cover physical status through questions about ability to perform daily living activities; (ii) comorbidity indices: the fact that HD patients suffer from several organ 
Table 1 Comparison of demographic and clinical variables between survivors and non-survivors

\begin{tabular}{|c|c|c|c|c|}
\hline Variables & Survivors & Non-survivors & Full sample & $\mathbf{P}$ \\
\hline \multicolumn{5}{|l|}{ Sex } \\
\hline Male & $84(60.0)$ & $14(66.6)$ & $98(60.9)$ & 0.637 \\
\hline Female & $56(40.0)$ & $7(33.4)$ & $63(39.1)$ & \\
\hline Age & $43.1 \pm 15.7$ & $53.6 \pm 11.9$ & $44.5 \pm 15.6$ & 0.002 \\
\hline \multicolumn{5}{|l|}{ Socioeconomic status ${ }^{a}$} \\
\hline B & $4(2.9)$ & $3(14.3)$ & $7(4.3)$ & \\
\hline C & $33(23.6)$ & $5(23.8)$ & 38 (23.6) & \\
\hline $\mathrm{D}$ & $81(57.9)$ & $13(61.9)$ & $94(58.4)$ & \\
\hline E & $22(15.7)$ & 0 & $22(13.7)$ & \\
\hline \multicolumn{5}{|l|}{ Primary kidney disease } \\
\hline Glomerulonephritis & $64(45.7)$ & $5(23.8)$ & $69(42.9)$ & 0.063 \\
\hline Hypertension & $31(22.1)$ & $7(33.4)$ & $38(23.6)$ & 0.276 \\
\hline Diabetes & $12(8.6)$ & $4(19.0)$ & $16(9.9)$ & 0.230 \\
\hline Policystic kidney & $9(6.4)$ & $2(9.5)$ & $11(6.8)$ & 0.117 \\
\hline Obstructive uropathy & $6(4.2)$ & $1(4.8)$ & $7(4.3)$ & 1.000 \\
\hline Lupus & $4(2.9)$ & 0 & $4(2.5)$ & 1.000 \\
\hline Chronic pyelonephritis & $4(2.9)$ & 0 & $4(2.5)$ & 0.658 \\
\hline Indeterminate & $10(7.2)$ & $2(9.5)$ & $12(7.5)$ & 1.000 \\
\hline Time on dialysis (months) & $55.4 \pm 52.8$ & $50.9 \pm 39.5$ & $54.8 \pm 51.2$ & 0.989 \\
\hline \multicolumn{5}{|l|}{ Comorbidity $^{\mathrm{b}}$} \\
\hline Low & $115(82.2)$ & $11(52.4)$ & $126(78.3)$ & 0.002 \\
\hline Medium & $22(15.7)$ & $6(28.6)$ & $28(17.4)$ & \\
\hline High & $3(2.1)$ & $4(19.0)$ & $7(4.3)$ & \\
\hline Included on transplant waiting list & $80(57.1)$ & $5(23.8)$ & $85(52.7)$ & 0.005 \\
\hline \multicolumn{5}{|l|}{ Laboratory tests } \\
\hline Creatinine (mg/dl) & $12.7 \pm 3.6$ & $11.8 \pm 4.0$ & $12.6 \pm 3.6$ & 0.140 \\
\hline Hemoglobin (g/dl) & $8.5 \pm 1.7$ & $8.2 \pm 1.6$ & $8.5 \pm 1.7$ & 0.665 \\
\hline Albumin $(\mathrm{g} / \mathrm{dl})$ & $4.3 \pm 0.4$ & $4.4 \pm 0.5$ & $4.3 \pm 0.4$ & 0.567 \\
\hline Calcium-phosphorus product $\left(\mathrm{mg}^{2} / \mathrm{dl}^{2}\right)$ & $46.2 \pm 13.0$ & $49.6 \pm 15.6$ & $46.6 \pm 13.4$ & 0.476 \\
\hline $\mathrm{Kt} / \mathrm{N}$ index & $1.5 \pm 0.5$ & $1.6 \pm 0.5$ & $1.5 \pm 0.5$ & 0.807 \\
\hline
\end{tabular}

Data are means $\pm S D$, or percentages (in parentheses)

aBrazilian Association of Research Institutes, B (best status) and E (worst status)

${ }^{\mathrm{b}}$ Khan index

dysfunctions besides kidney disease has made it usual in the field of nephrology to use comorbidity indices, like those of Khan, Davies and Charlson [20]; (iii) specific instruments related to renal disease severity: the use of the end-stage renal disease severity index (ESRD-SI) is another way of assessing physical status [21]; and (iv) the Karnofsky scale: the pioneer instrument on functional status is still useful [22].

The role of subjective measures must be remembered, even in a discussion about mortality, in which subjective measures did not predict death. First, in a scenario of high mortality, knowledge of how patients perceive their lives is a main outcome along with survival. Second, subjective feelings are influenced by cultural and socioeconomic status and for this reason there can be distinct patterns of samples. This point was the basis of our hypothesis that specific predictors of death among samples from low-income areas could be quite different from developed areas, a hypothesis not confirmed by our results. Only traditional and objective prognostic markers related to physical condition were identified.

The fact of only covering prevalent patients, hence including patients in different stages of disease and treatment, is a limitation of this study. But the finding that time on dialysis did not differ between survivors and non-survivors offsets this limitation, so that it does not preclude the hypothesis that the stage of treatment 
Table 2 Comparison of coping style and quality of life scores and prevalence of depression between survivors and non-survivors

\begin{tabular}{|c|c|c|c|c|}
\hline Variables & Survivors & Non-survivors & Full sample & $\mathbf{P}$ \\
\hline \multicolumn{5}{|l|}{ Coping style } \\
\hline Problem-oriented coping & $51.5 \pm 8.1$ & $50.2 \pm 10.8$ & $51.3 \pm 8.4$ & 0.332 \\
\hline Emotion-oriented coping & $67.9 \pm 9.5$ & $70.1 \pm 10.6$ & $68.2 \pm 9.7$ & 0.315 \\
\hline \multicolumn{5}{|l|}{ Quality of life } \\
\hline Physical functioning & $57.6 \pm 24.9$ & $42.6 \pm 26.3$ & $55.6 \pm 25.5$ & 0.023 \\
\hline Role-physical & $43.1 \pm 42.8$ & $46.4 \pm 39.7$ & $43.5 \pm 42.3$ & 0.667 \\
\hline Bodily pain & $60.6 \pm 30.5$ & $50.5 \pm 26.6$ & $59.3 \pm 30.2$ & 0.199 \\
\hline General health & $46.2 \pm 21.9$ & $44.1 \pm 18.9$ & $45.9 \pm 21.5$ & 0.523 \\
\hline Vitality & $56.7 \pm 23.5$ & $49.7 \pm 23.6$ & $55.8 \pm 23.6$ & 0.198 \\
\hline Social functioning & $70.6 \pm 29.4$ & $59.7 \pm 35.5$ & $69.2 \pm 30.3$ & 0.172 \\
\hline Role-emotional & $59.7 \pm 45.1$ & $54.0 \pm 46.5$ & $58.9 \pm 45.2$ & 0.487 \\
\hline Mental health & $64.8 \pm 21.7$ & $56.0 \pm 23.2$ & $63.7 \pm 22.0$ & 0.090 \\
\hline With depressive feelings & $11(7.8)$ & $2(9.5)$ & $13(8.0)$ & 0.679 \\
\hline
\end{tabular}

Data are means $\pm S D$, or percentages (in parentheses)

Table 3 Cox proportional hazards of death according to variables that differed between survivors and non-survivors

\begin{tabular}{cccc}
\hline Variable & Hazard ratio & 95\% confidence interval & P \\
\hline Comorbidity - low versus high risk $^{\mathbf{a}}$ & 8.958 & $2.843-28.223$ & $<0.001$ \\
\hline Not being on the transplant waiting list $_{\text {Age }}$ & 3.992 & $1.462-10.902$ & 0.007 \\
\hline Physical functioning $^{\mathbf{b}}$ & 1.038 & $1.012-1.066$ & 0.005 \\
\hline
\end{tabular}

${ }^{\mathrm{a}}$ Khan index

${ }^{\mathrm{b}} \mathrm{SF}-36$

and disease is less important to predict risk of death than physical status. And above all, the method usually used in dialysis studies about mortality is the same as that presented here: yearly crude mortality rate considering prevalent patients. Moreover, patients with fewer than three months on treatment were excluded to avoid confusion of clinical instability and poor physical status, typical of incidental patients undergoing HD. Another important limitation is the small size of the sample and low number of deaths. The reason is that the study comprised patients from a single renal unit. But we must highlight that this renal unit is the only one covering a wide region and the only place where end-stage renal disease patients are treated in that region, so the data have the potential to validate the sample as characteristic of northeastern Brazil.

\section{Conclusions}

The variables that were able to indicate risk of death were all related to physical status, like age, comorbidity grade and the condition of not being on the transplant waiting list. The only of quality of life dimension-a possible subjective indicator-that was correlated with death was physical functioning, which reflects physical status more than subjective feelings.

\section{Abbreviations}

ESRD: End-stage renal disease; HD: Hemodialysis; SF-36: Medical Outcomes Study 36-Item Short Form Health Questionnaire.

\section{Authors' contributions}

PRS was involved in conceptualizing the study, collecting data and writing the manuscript.

\section{Competing interests}

The authors declare that they have no competing interests.

Received: 31 October 2011 Accepted: 11 January 2012

Published: 11 January 2012

\section{References}

1. Quinton W, Dillard D, Scribner BH: Cannulation of blood vessels for prolonged hemodialysis. Trans Am Soc Artif Intern Organs 1960, 6:104-113.

2. Brazilian Society of Nephrology: Censo.[http://www.sbn.org.br/censos/ censos_anteriores/censo_2008.pdf].

3. Himmelfarb J: Uremic toxicity, oxidative stress, and hemodialysis as renal replacement therapy. Semin Dial 2009, 22:636-643.

4. Goodkin DA, Young EW, Kurokawa K, Prutz KG, Levin NW: Mortality among hemodialysis patients in Europe, Japan, and the United States: case-mix effects. Am J Kidney Dis 2004, 44:16-21.

5. Sesso R: Epidemiologia da doença renal crônica no Brasil e sua prevenção.[http://www.cve.saude.sp.gov.br/htm/cronicas/irc_prof.htm] 
6. Santos PR: Qualidade de vida relacionada à saúde entre pacientes de baixa renda em hemodiálise. In Atualidades em nefrologia. Volume 11. Edited by: Cruz J. São Paulo: Sarvier; 2010:428-434.

7. Santos PR: Associação de qualidade de vida com hospitalização e óbito em pacientes portadores de doença renal crônica em hemodiálise. $J$ Bras Nefrol 2005, 27:184-190.

8. Cicconelli RM, Ferraz MB, Santos W, Meinão I, Quaresma MR: BrazilianPortuguese version of the SF-36: a reliable and valid quality of life outcome measure. Rev Bras Reumatol 1999, 39:143-150.

9. Jalowiec A, Powers MJ: Stress and coping in hypertensive and emergency room patients. Nurs Res 1981, 30:10-15.

10. Souza-Talarico JN, Chaves EC, Nitrini R, Caramelli P: Stress and coping in older people with Alzheimer's disease. J Clin Nurs 2009, 18:457-465.

11. Lazarus RS, Folkman S: Stress, appraisal, and coping. New York: Springer Publishing; 1984.

12. Andresen EM, Malmgren JA, Carter WB, Patrick DL: Screening for depression in well older adults: evaluation of a short form of the CES-D (Center for Epidemiologic Studies Depression Scale). Am J Prev Med 1994, 10:77-84.

13. Brazilian Association of Research Institutes: Critério Brasil.[http://www. marketanalysis.com.br/arquivos_download/biblioteca/cceb-1.pdf].

14. Khan IH, Campbell MK, Cantarovich D, Catto GRD, Edward N, Fontenaille C, Fleming LW, Gerlag PGG, Van Hamersvelt HW, Henderson IS, Koene RAP, Papadimitriou M, Ritz E, Russel IT, Stier E, Tsakiris D, MacLeod AM: Survival on renal replacement therapy in Europe: Is there a "centre effect". Nephrol Dial Tranplant 1996, 11:300-307.

15. Daugirdas JT: Second generation logarithmic estimates of single-pool variable volume Kt-V: an analysis of error. J Am Soc Nephrol 1993, 4:205-213.

16. Revuelta KL, López FJG, Moreno FA, Alonso J: Perceived mental health at the start of dialysis as a predictor of morbidity and mortality in patients with end-stage renal disease. Nephrol Dial Transplant 2004, 19:2347-2353.

17. Lopes AA, Bragg J, Young E, Goodkin D, Mapes D: Depression as a predictor of mortality and hospitalization among hemodialysis patients in the United States and Europe. Kidney Int 2002, 62:199-207.

18. Lowrie EG, Curtin RB, Lepain N, Schatell D: Medical Outcomes Study Short Form-36: a consistent and powerful predictor of morbidity and mortality in dialysis patients. Am J Kidney Dis 2003, 41:1286-1292.

19. Rai M, Rustaqi T, Rustaqi S, Kohli R: Depression, insomnia and sleep apnea in patients on maintenance hemodialysis. Indian J Nephrol 2011, 21:223-229.

20. Van Manen JG, Korevaar JC, Dekker FW, Boeschoten EW, Bossuyt PMM, Krediet RT: Adjustment for comorbidity in studies on health status in ESRD patiens: Which comorbidity index to use. J Am Soc Nephrol 2003, 14:478-485.

21. Craven J, Littlefield C, Rodin G, Murray M: The end-stage renal disease severity index (ESRD-SI). Psychol Med 1991, 21:237-243.

22. Schag CC, Heinrich RL, Ganz PA: Karnofsky performance status revisited: Reliability, validity, and guidelines. J Clin Oncology 1984, 2:187-193.

doi:10.1186/1756-0500-5-24

Cite this article as: Santos: Evaluation of objective and subjective indicators of death in a period of one year in a sample of prevalent patients under regular hemodialysis. BMC Research Notes 2012 5:24.

\section{Submit your next manuscript to BioMed Central and take full advantage of:}

- Convenient online submission

- Thorough peer review

- No space constraints or color figure charges

- Immediate publication on acceptance

- Inclusion in PubMed, CAS, Scopus and Google Scholar

- Research which is freely available for redistribution 